\title{
Do Gradations of Time Zone Separation Make a Difference in Performance? A First Laboratory Study
}

\author{
J. Alberto Espinosa \\ American University \\ alberto@american.edu
}

\author{
Ning Nan \\ University of Oklahoma \\ nnan@ou.edu
}

\author{
Erran Carmel \\ American University \\ carmel@american.edu
}

\begin{abstract}
We often hear that global software engineering teams are affected by time differences. While there is considerable research on the difficulties of distance, culture and other dimensions, there has been little research that isolated the impact of just time differences. The research question that guides us is whether there are gradual differences across time zones that impact team performance. In this study we conducted a laboratory experiment with 42 dyadic teams. The teams were randomly assigned into 4 time zone overlap conditions: full overlap, $2 / 3$ overlap, 1/3 overlap and no overlap. Using a fictional map task, we found that participants' perceptions of process are unrelated to actual objective performance measures of speed and accuracy. Consistent with our expectations, we found that a small time separation has no effect on accuracy, but that more time separation has a significant effect on accuracy. Also consistent with our expectations, we found that a small amount of time separation has a significant effect on production speed. However, contrary to our expectations, we found that further increases in partial overlap have less significant effects on speed, and when there is no overlap speed actually increases, albeit not significantly-a "U-shaped" effect.
\end{abstract}

\section{Introduction}

It is generally accepted that time zone differences negatively impact work in global software engineering. While there is a plethora of research on the difficulties of distance, culture and other dimensions [1-3, 9], the study of time separation has gone largely neglected. With time-separated collaboration, the lack of overlap time means less synchronous coordination time. The result is likely to be a negative impact on project outcome measures.

However, research has not yet answered this key question for global software engineering: assuming everything else constant, are there measurable differences between working with a teammate that is 3 time zones versus 11 time zones away. From the perspective of time zones alone, if one is in New York, is it really better to source to Mexico than India? Therefore, the research question that guides us is whether there are gradual differences across time zones that impact team performance.

In this paper we present preliminary results of a controlled laboratory experiment in which we examined this research question - a first of its kind. We set up teams that worked with full time overlap, significant time overlap, some time overlap, and no time overlap. We collected survey data and measured task outcomes. In this first paper we focus on the main dependent variables that we measured: speed and accuracy.

\section{Theory and Hypotheses}

Since the 1990s there has been considerable research that compares co-located to electronicmediated teams [12] and the effect of media richness [5], followed by several empirical studies. This large body of literature generally shows that in spite of the improvement in technologies, electronic-mediated teams do not usually overcome the benefits of synchronous work and co-presence (i.e., the ability to get immediate clarification on an issue; frequent and spontaneous interaction, availability of contextual reference, presence awareness, workspace awareness, etc.). However, to the best of our knowledge, no prior studies have investigated how time separation affects team performance. Given that distance and time separation are often correlated in global collaboration, and given today's increased offshore outsourcing practices [4] and global collaboration across time zones [9], it is important that we develop a better understanding of how time separation affects team performance.

While time separation can be caused by several reasons (e.g., difference in work schedules, mobile 
workforce, differences in holidays and vacation), we focus more specifically on the effect of time zones. There is considerable research on teams that are either in the same time zone or many time zones apart (but without controlling for time zone differences). But these two contexts are insufficient to understand the effect of time zones. There have been some tentative conceptual steps in this direction: Griffith et al. [8] proposed "percentage of time apart in the task" as one of the dimensions of "virtualness". Kirkman and Mathieu [10] took this further and proposed "synchronicity" to distinguish between "real-time" (i.e., synchronous) and "lagged-time" (asynchronous) interaction. O'Leary and Cummings [11] measured time separation mathematically using an overlap index of the fraction of the work day that overlaps between two sites. At the same time, O'Leary and Cummings observed that distance and time separation could be represented in various configurations, depending on how many sites are involved and how team members are distributed across sites.

Studying the effects of time zones more precisely would be very difficult if too many different time zone configurations are represented in the team sample. Espinosa and Carmel [6] formulated a dyadic model of coordination across time zones to better understand the challenges that two members separated by time zones face. They argued that in order to understand how work is carried out across time zones one first needs to understand how time separation affects a single task carried out by one dyad with a one-way dependency. In their model, there are two actors, a task requestor $(\boldsymbol{R})$ and a task producer $(\boldsymbol{P})$ with a single task at hand and the work of $\boldsymbol{R}$ depends on $\boldsymbol{P}$ completing the requested task. This model suggests: (1) that there are inherent delays due to time separation in that $\boldsymbol{P}$ may be producing while $\boldsymbol{R}$ is sleeping (i.e., negative delay) or that $\boldsymbol{R}$ has to wait because $\boldsymbol{P}$ is sleeping when the request was sent (i.e., positive delay); and (2) that further delays can be incurred due to miscommunication that requires either further communication or even re-work. Repairing such miscommunication is costly in time separated environments because one often has to wait until $\boldsymbol{P}$ starts his or her workday. Therefore, we posit that as the work time overlap is reduced, it takes longer for a team to complete a task:

H1: as time difference increases (i.e., work time overlap is reduced) between two sites, production speed will decrease.

Another important outcome measure is quality or accuracy in the execution of the task. To the best of our knowledge, there are no prior empirical studies that have investigated how time separation affects accuracy or task quality. From our arguments above, we anticipate that teams with more time separation will have more difficulty identifying and repairing miscommunication on a timely manner because their communication is more asynchronous and less interactive. Because teams often have time pressure to complete a task, they may overlook mistakes made by their teammates, or they may consciously decide to trade quality in favor of production speed, depending on the economic incentives for one or the other. Thus, we posit:

H2: as time difference increases (i.e., work time overlap is reduced) between two sites, production quality (i.e., accuracy) will decrease.

\section{Experimental Design}

To test the hypotheses, we conducted a laboratory experiment varying the degree of time zone overlap (full time overlap, 2/3 time overlap, 1/3 time overlap and no time overlap). A total of 84 subjects participated in our experiment. The participants were recruited from three universities in the U.S., but participants were not limited to students. Participants' ages ranged from less than 21 to over 40 . The gender distribution was almost evenly split between males and females. Education levels ranged from high school (in college) to master's degree. Participants were randomly arranged into dyadic teams. The dyadic teams were assigned to one of the four time zone overlap conditions. The study design resulted in 10 dyads in the full overlap condition, 12 dyads in the $2 / 3$ overlap condition, 10 dyads in the $1 / 3$ overlap condition and 10 dyads in the no overlap condition. Participants were paid $\$ 20$ for their participation in the experiment and a performance bonus of $\$ 40$ for each member of the best performing team in each condition (based on speed and accuracy).

Because our goal was to better understand how software teams work across time zones, our initial inclination was to use a software task. We decided in favor of a fictional map task instead to eliminate possible confounds due to differences in the software programming abilities of the participants. We adopted a modified version of the fictional map task because it has been used in previous experimental studies [14] and because it requires the type of knowledge workflow information that needs to be exchanged in a software task. The maps had to be built with digital components (i.e., graphic backgrounds, icons, arrows) 
we had provided to the map producer $\boldsymbol{P}$ based on the requirements articulated by the map requestor $\boldsymbol{R}$.

In this task, one subject of each dyadic team played the role of a map designer (i.e., the requestor $\boldsymbol{R}$ ) and the other played the role of a map maker (i.e., the producer $\boldsymbol{P}$ ). This role is similar to that of a software designer who needs to communicate design specifications to a programmer. Each map designer had a set of 13 maps (see figure 1 for an example) while each map maker had a set of 13 blank PowerPoint $($ C slides. Figure 1 shows an example of a map. The first map was for practice and training and the remaining 12 maps were for the actual task. A map designer's task was to give instructions to the map makers about how to replicate the maps.

A map maker's role was to reproduce the maps in the PowerPoint slides based on the instructions. We provided the map makers with a PowerPoint file that contained all the necessary elements to draw the maps. Thus, rather than drawing a map from scratch, a map maker needed to identify the correct elements and insert them into the blank slides at the correct location. The fictional map task mimics important aspects of global software engineering teams, including: (1) shared goals; (2) interdependent activities and skills; (3) the need for effective communication; and (4) the need to articulate and interpret requirements correctly.

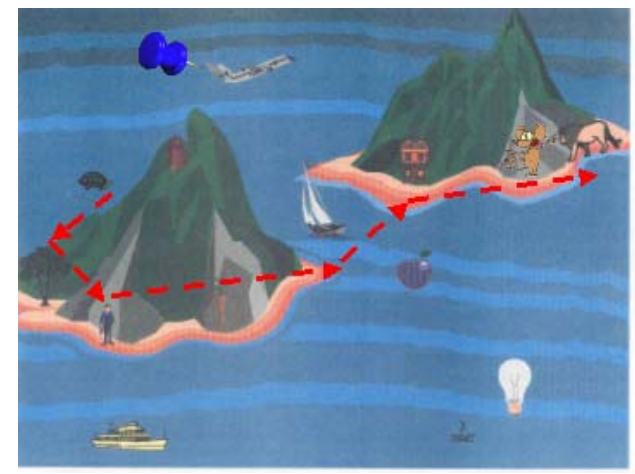

Figure 1: An Example of a Map

Each experimental session included four synthetic workdays. Each workday lasted for 15 minutes with a 5 minute break (i.e., off work hours) between workdays. In the partially overlap conditions, map designers would start workdays first and then followed by map makers. During the experiment, participants were only allowed to communicate using a chat software tool that we provided to simulate geographically distributed collaboration, eliminate confounds with distance, and capture the team's interaction. Participants didn't know who their teammates were. Using a single communication channel also helped us rule out potential confounding effects of media richness. During the overlap time, subjects could "chat" with their teammates whenever they wanted. On the other hand, during the nonoverlap time, subjects could add instructions or comments through the chat facility, but they had to wait until their colleagues came to work to receive a response.

The objective outcome measures of the experiment included speed and accuracy. Specifically, speed is indicated by the number of maps a team could replicate divided by the total number of possible maps (i.e., 12) to normalize the scale from 0 to 1 . Accuracy was measured for each map simply by counting the number of correct elements (backgrounds, icons and arrows) in the map and their correct position in the picture. The score was computed by counting the number of correct elements and positions and dividing this by the number of correct elements and positions for a perfect map (i.e., 15), to normalize the scale from 0 to 1 , and then averaged for all the team's maps. Speed and accuracy metrics for each map were evaluated independently by an external rater and one of the experimenters with a reliability of $90.5 \%$. The differences between these two raters were reconciled by another experimenter.

At the end of the experiment, participants completed a survey to gather self-report data on perceptions of process and outcomes (i.e., communication, coordination processes and coordination outcomes). In addition to demographic data, we collected a total of 25 survey items. These items were reduced using factor analysis with Varimax rotation, which yielded 6 factors explaining $68.7 \%$ of the total variance. Items with a factor loading greater than 0.5 were grouped with that factor. We discarded 4 survey items that didn't have any factor loadings of 0.5 or greater. We also discarded the 6th factor because it contained a single survey item of little interest to the study, reducing our variable set to 20 survey items and 5 factors explaining $64.2 \%$ of the total variance. The factor groups were tested for reliability and all variables had a Cronbach- $\alpha$ reliability of 0.7 or greater.

We constructed five variables with this procedure measuring perceptions of: (1) communication quality (e.g., "I received accurate [e.g. correct or precise] information from my teammate"); (2) mechanistic coordination (i.e., use of non-communication means to coordinate activities in a programmed way - e.g., "we established ground rules, routines and/or procedures to facilitate our team's work"); (3) communication delay (e.g., "typically it took a long time to get a response from my teammate"); (4) miscommunication problems 
(e.g., "our communication with my teammate required frequent clarification"); and (5) coordination process problems (e.g., "we had many problems due to confusion and misunderstanding [by me or my teammate] about our task requirements"). The descriptive statistics and correlation matrix for all the study variables are displayed in Table 1.

\section{Data Analysis}

While we found interesting differences in key variable means across time separation conditions, an ANOVA test of means did not find significant differences for most key variables. This was surprising, but a further look into the data revealed some important nuances. We further analyzed the data using regression analysis.

Because our observations during the experimental session and the chat logs revealed substantial variation in performance within conditions, we included control variables in all our models to account for factors that may affect speed and accuracy, including: (1) research site - using a binary variable for each site; (2) age group; (3) gender; (4) educational level; and (5) team member ratings of skills (for self and the teammate). Time separation was modeled with a separate binary variable for each of the 4 conditions. The full overlap binary variable was left out of the model as the control (i.e., the intercept) to avoid the dummy variable trap (i.e., perfect linear dependency when all 4 variables are included).

We first regressed all control and time separation variables on communication quality and mechanistic coordination. In a structural fashion (for non-recursive models), we then regressed these variables, plus communication quality and mechanistic coordination on communication delay, miscommunication problems and coordination process problems. Finally, we regressed all these variables, plus miscommunication problems and coordination process problems on speed and accuracy. In every model that included process variables, we retained all control and time separation variables, but removed non-significant process variables to preserve degrees of freedom. Removal of these variables was justified with a Wald's F statistic that tested if the block of variables removed did not affect the predictive power of the respective regression model. This type of modeling allowed us to compare perceptions by participants with objective measures of performance. The results of our regression models are presented in Table 2.

\section{Results}

\subsection{Control Variables}

Older age groups had more miscommunication and coordination process problems, and marginally lower accuracy, but there was no difference in speed. Team rated skills had a positive association with communication quality and a negative association with communication delay. Gender and education level had no effects on any variables.

\subsection{Effect of Time Separation on Team Process}

We first analyzed how time separation affected team member responses to survey items about team process. The corresponding regression results are presented in the first 5 regression models in Table 2. Compared to full overlap (i.e., the control condition), there were no differences in communication quality with the partial overlap condition, but teams in the no overlap condition perceived lower levels of communication quality. Teams in all time separated conditions exhibited more mechanistic coordination than teams in the full overlap condition but, surprisingly, this difference was only marginally significant for teams in the $1 / 3$ overlap condition and non-significant for the other two conditions. Teams in the no overlap condition reported more communication delay than teams with full overlap but none of the partial overlap conditions had significant differences with full overlap. Time separation had no effect on miscommunication problems but it increased process coordination problems with little (i.e., 1/3 overlap) or no overlap.

\subsection{Effect of Time Separation on Performance}

Regression results on speed and accuracy are presented in the last 2 regression models in Table 2 .

Speed. Interestingly, disconfirming our first hypothesis, compared to full overlap, there was no difference in speed with the no overlap condition, but speed decreased significantly with very small amounts of time separation (i.e., 2/3 overlap). Similarly, teams in the $1 / 3$ overlap had lower levels of speed than full overlap, but the difference was less significant than for $2 / 3$ overlap. Surprisingly, teams in the no overlap condition had higher speed than full overlap but this difference was not significant (note that the p-value was very close to becoming significant). 


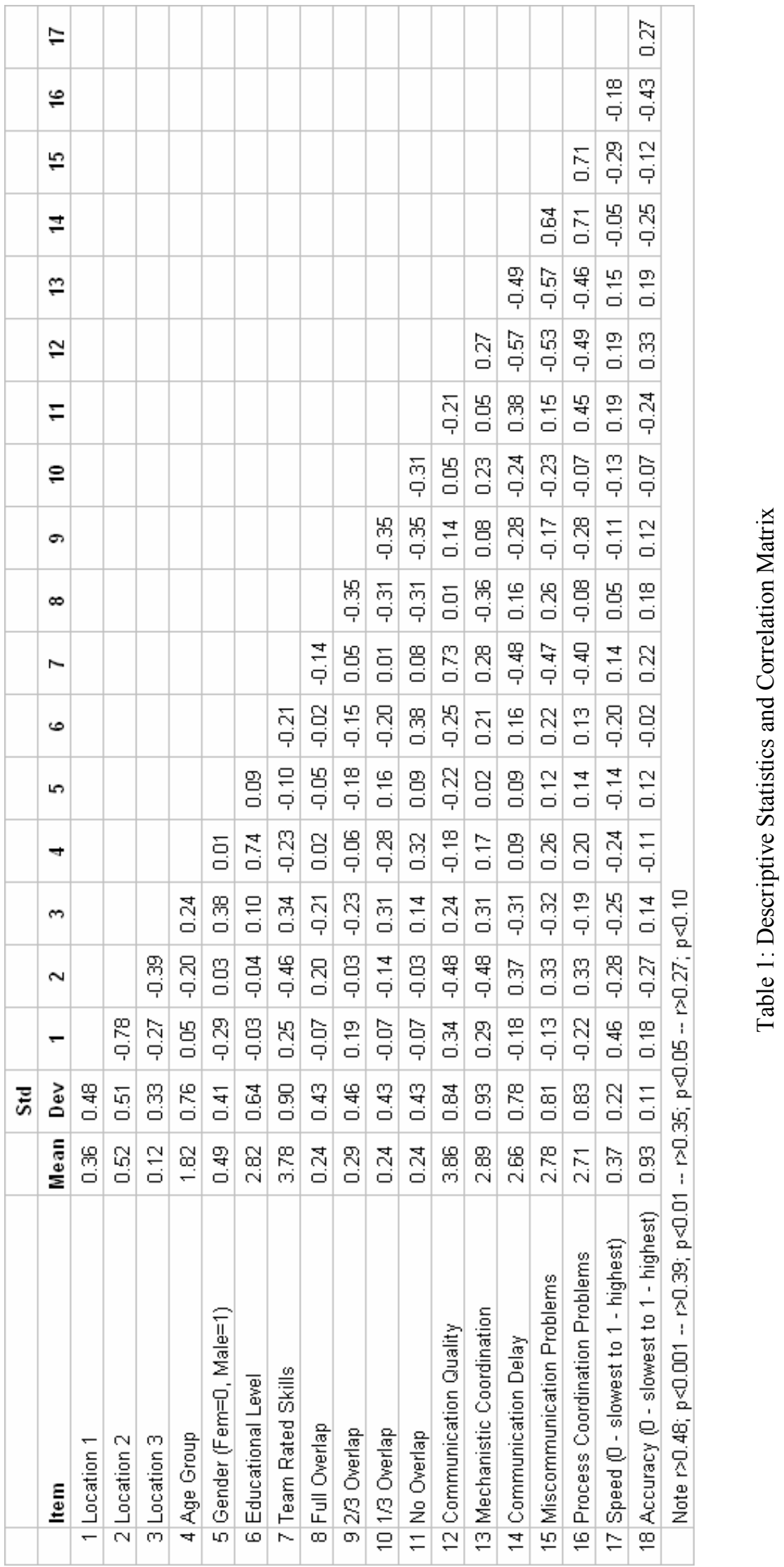




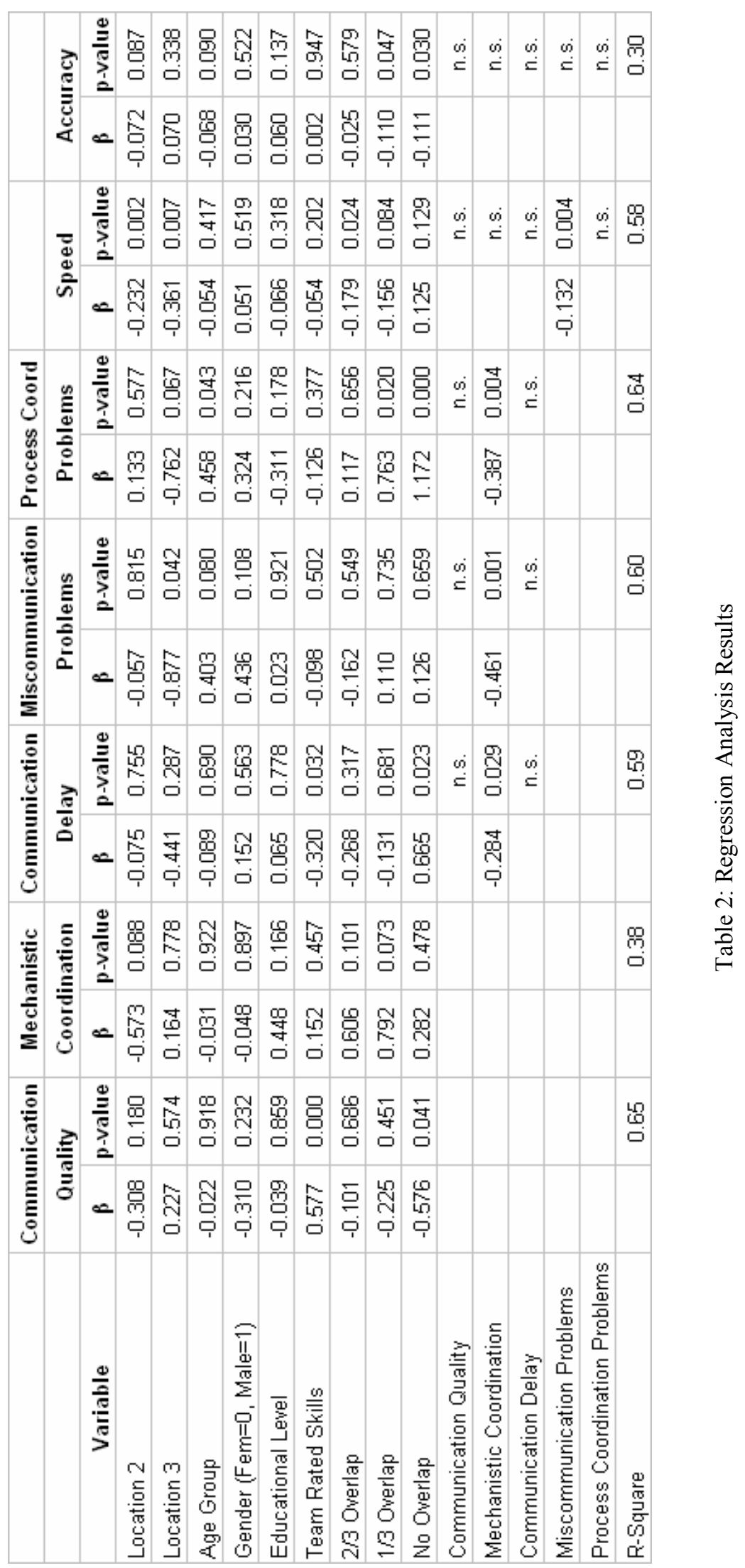


Accuracy. As we anticipated in our second hypothesis, teams with more time separation (i.e., 1/3 and no overlap) had significantly lower levels of accuracy, but a small amounts of time separation (i.e., $2 / 3$ overlap) had no effect on accuracy.

\subsection{Process Variables and Performance}

Process variables provide interesting insights into team dynamics because they measure team members' perceptions of what is happening. Surprisingly, perceived communication quality did not have an effect in any of the models. On the other hand, perceived mechanistic coordination had a significant negative association with communication delay, miscommunication problems, and process coordination problems, underscoring the importance of mechanistic coordination in time separated software tasks. Perceived communication delay and process coordination problems had no effects with any variable, but perceived miscommunication problems had a strong negative association with speed.

\section{Discussion}

Upon first inspection of the means of our main dependent variables we found some variation across time zones, but it was not significant. However, upon further inspection controlling for variables that could affect speed and accuracy, we found some interesting effects. This suggests that simply comparing performance variables across conditions may lead to incorrect conclusions. As illustrated in Figure 2, our results suggest a "U" shaped effect of time separation on speed and a negative effect on accuracy. In addition, our results also suggest a disconnect between team members perceptions of communication quality, communication delay and process coordination problems, and objective measures of speed and accuracy. The no overlap condition provided very interesting and somewhat surprising results. While no overlap was associated with lower perceived levels of communication quality, higher communication delay and process coordination problems, it had no impact on speed, compared to full overlap. Team members perceived that the lack of overlap affected their ability to work fast, but when their actual speed was compared to that of full overlap teams, they were actually able to work faster.

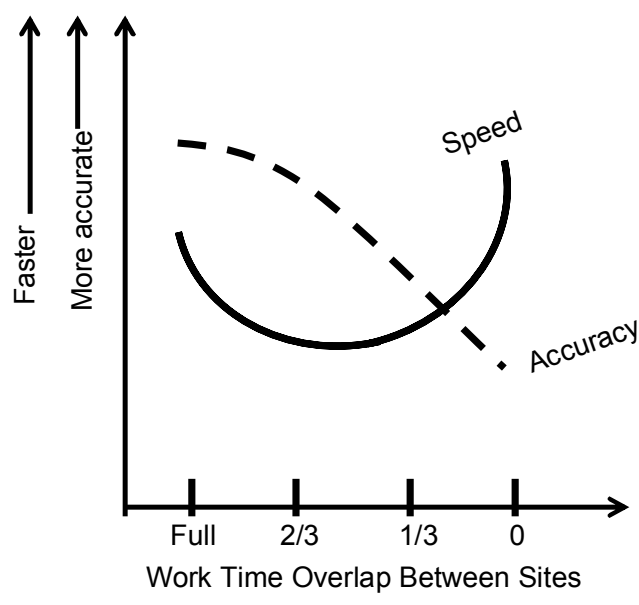

Figure 2: The Time Zone Effects on Speed and Accuracy

In contrast, teams with a small partial overlap (i.e., $1 / 3$ ) experienced more process coordination problems, but as the partial overlap increased (i.e., to 2/3) the problems disappeared. Surprisingly, none of the partial overlap conditions had an effect on team member perceptions of either communication delay or miscommunication problems. However, both partial overlap conditions (i.e., $1 / 3$ and $2 / 3$ ) were associated with lower levels of speed and accuracy but, interestingly, accuracy was more significantly affected by larger time separation (i.e., 1/3 overlap), whereas speed was more significantly affected by small time separation (i.e., 2/3). These results suggest that the frustrations experienced by team members when working across time zones may not necessarily translate into actual declines in performance levels.

Furthermore, and perhaps the most interesting result in our study, as the work time overlap was reduced (from full to $2 / 3$ ) there was a substantial reduction in speed, but that as overlap was further reduced to $1 / 3$ the negative effect on speed weakened, and when the work overlap was totally eliminated, teams actually worked faster. We attribute this surprising "U" shape effect of time separation on speed to the fact that the task is relatively simple, so teams can work efficiently and uninterrupted when they have no overlap and can articulate and interpret instructions clearly. However, when teams need to constantly change their interaction mode from synchronous to asynchronous they need to make mental adjustments in their work styles and coordination tactics, thus reducing their efficiency. We speculate that with more complex and equivocal tasks that require more frequent interaction, speed will be dramatically affected by time separation, but this effect may also be influenced by learning effects.

In contrast, small reductions in work time overlap had no effect on accuracy, perhaps because team 
members had sufficient windows for synchronous interaction in which they could resolve miscommunication issues. However, the negative effect of time separation on accuracy became more significant as the work time overlap was further reduced and then eliminated. These distinct effects (U shaped for speed vs. diminishing for accuracy) are consistent with our speculation that time separation has some effect on real communication and misunderstandings, albeit unnoticed, which negatively affect accuracy and could therefore negatively affect speed with more complex and equivocal tasks.

\section{Implications}

We find our results interesting and somewhat surprising. The main implication for practice is that there are tradeoffs on the effect of time separation between speed and accuracy and managers need to evaluate this very carefully when making work assignments and outsourcing decisions based on geography, depending on the goals of the software project. Partial time separation has detrimental effects on speed because teams need to learn and adjust to this dual interaction mode (i.e., synchronous-asynchronous) and monitor time differences carefully, but it has little or no effect when time separation is large. In contrast, output quality (i.e., accuracy) does not suffer with small amounts of time separation, but it is substantially affected as time separation is increased.

Our results on mechanistic coordination also have important practical implications for team members working in highly interdependent software tasks that require substantial coordination. Except for the fact that teams with a small work time overlap exhibited a marginally larger use of mechanistic coordination practices, nothing else predicted the use of mechanistic coordination. At the same time, mechanistic coordination had the strongest effect on reduced perceived communication delay, miscommunication problems, and process coordination problems. While only miscommunication problems had a negative effect on one performance measure (i.e., speed), we anticipate that reduced communication delay, miscommunication problems and process coordination problems will become critical to both, speed and accuracy as the task activities become more interdependent, requiring more coordinated effort.

These results are consistent with other studies conducted with real organizations, which found that mechanistic coordination is very important in time separated contexts, but that team members select coordination mechanisms based mostly on personal preferences and not so much on geographic factors [7].
But this and prior research provide strong evidence that time separated teams that organize their tasks effectively (i.e., using mechanistic coordination) can better offset the problems of untimely and limited communication in more asynchronous conditions and reduce problems of miscommunication, thus helping them perform at higher levels. While the well established coordination theory prescribes that mechanistic coordination is more important for more certain routine tasks [13], our research provides important evidence that mechanistic coordination is also important when coordination via communication is hindered by geographic barriers.

Our study also has interesting implications for research. No prior study has teased out the effects of time separation from geographic distance or media richness. Our controlled experimental environment has enabled us to learn the nuances of working across time boundaries. Naturally, our study has limitations, but these limitation offer interesting possibilities for further research. While we can think of many possible extensions to this study, the ones we are planning in the near future include: (1) manipulating the equivocality or complexity of the task - we anticipate that the effects of time separation will be different and perhaps more dramatic for more complex and more equivocal tasks that require more frequent interaction; (2) manipulating the nature of dependencies - with the one-way sequential dependency used in our experimental task, teams can adjust and learn to batch and process task requests; we anticipate that the effects of time separation will be stronger with more interdependent tasks; (3) manipulating training conditions - we speculate that the lower performance in partial overlap conditions will disappear if team members are trained to work in this dual mode condition; and (4) we need to extend this study to larger teams working in various geographic configurations in which more than two team members are working from various locations and time zones.

\section{Acknowledgements}

This research was funded by the Center for Information Technology and the Global Economy (CITGE) at the Kogod School of Business at American University, Washington, D.C. and Dean's Discretionary Fund from Horace H. Rackham School of Graduate Studies at University of Michigan.

\section{References}

[1] Ahuja, M.K., D.G. Galletta, F., and K. Carley, Individual Centrality and Performance in Virtual R\&D Groups: an 
Empirical Study. Management Science, 2003. 49(1): p. 2138.

[2] Armstrong, D.J. and P. Cole, Managing Distances and Differences in Geographically Distributed Work Groups, in Distributed Work, P. Hinds and S. Kiesler, Editors. 2002, MIT Press: Cambridge, MA. p. 187-215.

[3] Carmel, E., Global Software Teams. 1999, Upper Saddle River, NJ: Prentice Hall.

[4] Carmel, E. and P. Tjia, Offshoring Information Technology: Sourcing and Outsourcing to a Global Workforce. 2005, Cambridge, U.K.: Cambridge University Press.

[5] Daft, R. and R. Lengel, Organizational Information Requirements, Media Richness and Structural Design. Management Science, 1986. 32(5).

[6] Espinosa, J.A. and E. Carmel, The Impact of Time Separation on Coordination in Global Software Teams: a Conceptual Foundation. Journal of Software Process: Practice and Improvement, 2004. 8(4): p. 249-266.

[7] Espinosa, J.A. and C. Pickering. The Effect of Time Separation on Coordination Processes and Outcomes: A Case Study. in 39th Hawaiian International Conference on System Sciences. 2006. Poipu, Kauai, Hawaii: IEEE.
[8] Griffith, T.L., J.E. Sawyer, and M.A. Neale, Virtualness and Knowledge in Teams: Managing the Love Triangle of Organizations, Individuals, and Information Technology. MIS Quarterly, 2003. 27(2): p. 265-287.

[9] Herbsleb, J.D. and A. Mockus, An Empirical Study of Speed and Communication in Globally Distributed Software Development. IEEE Transactions on Software Engineering, 2003. 29(6): p. 481-494.

[10] Kirkman, B.L. and J. Mathieu, The Dimensions and Antecedents of Team Virtuality. Journal of Management, 2005. 31(5): p. 1-19.

[11] O'Leary, M.B. and J.N. Cummings, The Spatial, Temporal, and Configurational Characteristics of Geographic Dispersion in Teams. MIS Quarterly, 2007. 31(3).

[12] Sproull, L. and S. Kiesler, Connections: New Ways of Working in the Networked Organization. 1991, Cambridge, MA: MIT Press.

[13] Van de Ven, A.H., L.A. Delbecq, and R.J. Koenig, Determinants of Coordination Modes Within Organizations. American Sociological Review, 1976. 41(2): p. 322-338.

[14] Veinott, E.S., et al. Video Helps Remote Work: Speakers Who Need to Negotiate Common Ground Benefit from Seeing Each Other. in CHI 99. 1999. 